\title{
Artificial Chromosomes to Explore and to Exploit Biosynthetic Capabilities of Actinomycetes
}

\author{
Rosa Alduina and Giuseppe Gallo \\ Department of Science and Molecular and Biomolecular Technology, University of Palermo, Viale delle Scienze, 90128 Palermo, Italy \\ Correspondence should be addressed to Giuseppe Gallo, valeria.alduina@unipa.it
}

Received 5 April 2012; Revised 20 June 2012; Accepted 4 July 2012

Academic Editor: Jozef Anné

Copyright ( $) 2012$ R. Alduina and G. Gallo. This is an open access article distributed under the Creative Commons Attribution License, which permits unrestricted use, distribution, and reproduction in any medium, provided the original work is properly cited.

\begin{abstract}
Actinomycetes are an important source of biologically active compounds, like antibiotics, antitumor agents, and immunosuppressors. Genome sequencing is revealing that this class of microorganisms has larger genomes relative to other bacteria and uses a considerable fraction of its coding capacity (5-10\%) for the production of mostly cryptic secondary metabolites. To access actinomycetes biosynthetic capabilities or to improve the pharmacokinetic properties and production yields of these chemically complex compounds, genetic manipulation of the producer strains can be performed. Heterologous expression in amenable hosts can be useful to exploit and to explore the genetic potential of actinomycetes and not cultivable but interesting bacteria. Artificial chromosomes that can be stably integrated into the Streptomyces genome were constructed and demonstrated to be effective for transferring entire biosynthetic gene clusters from intractable actinomycetes into more suitable hosts. In this paper, the construction of several shuttle Escherichia coli-Streptomyces artificial chromosomes is discussed together with old and new strategies applied to improve heterologous production of secondary metabolites.
\end{abstract}

\section{Introduction}

Actinomycetes, Gram-positive bacteria, represent an important source of biologically active compounds. In fact, they synthesize one-third of the antibiotics commercially available - that is, the macrolide erythromycin, the glycopeptide vancomycin, and the cyclic lipopeptide daptomycinand other drugs like neuroprotectants (e.g., meridamycin), and anticancer compounds (e.g., migrastatin or geldanamycin) [1]. Most natural products possess complex structures (Figure 1) that are very difficult and expensive to be chemically synthesized and they are obtained at an industrial scale mainly by fermentation processes from the producer microorganism. However, industrial production requires microbial strains that produce unnaturally high yields of a secondary metabolite. In many cases, industrial fermentation yields more than $10 \mathrm{~g} / \mathrm{L}$ of product; that is, penicillin production process was steadily improved over the years to allow titres of $70 \mathrm{~g} / \mathrm{L}$ [2]. To increase production yields, classical strain improvement (CSI) or rational genetic methods are used. CSI consists of sequential random mutagenesis and screening of the mutant strains: after each cycle of CSI a population of improved mutants is identified from which the single best performer is selected. CSI does not give information on genes and molecular mechanisms during the improvement and it is likely that some mutations may be detrimental or neutral in term of production.

Rational methods consist of deleting or overexpressing specific genes that, finally, control biosynthesis of a secondary metabolite [3], relying on the knowledge of gene sequences and functions and on suitable protocols for the genetic manipulation of natural producers. The biosynthetic genes devoted to secondary metabolite biosynthesis are organised in clusters that can occasionally reach $100 \mathrm{~kb}$ in size or more $[4,5]$. Since suitable protocols for the genetic manipulation of natural producers are not always available and given the size of most gene clusters, artificial chromosome-based vectors that can be maintained in Streptomyces have been constructed. Such vectors allow genetic information for secondary metabolite production to be transferred from the 


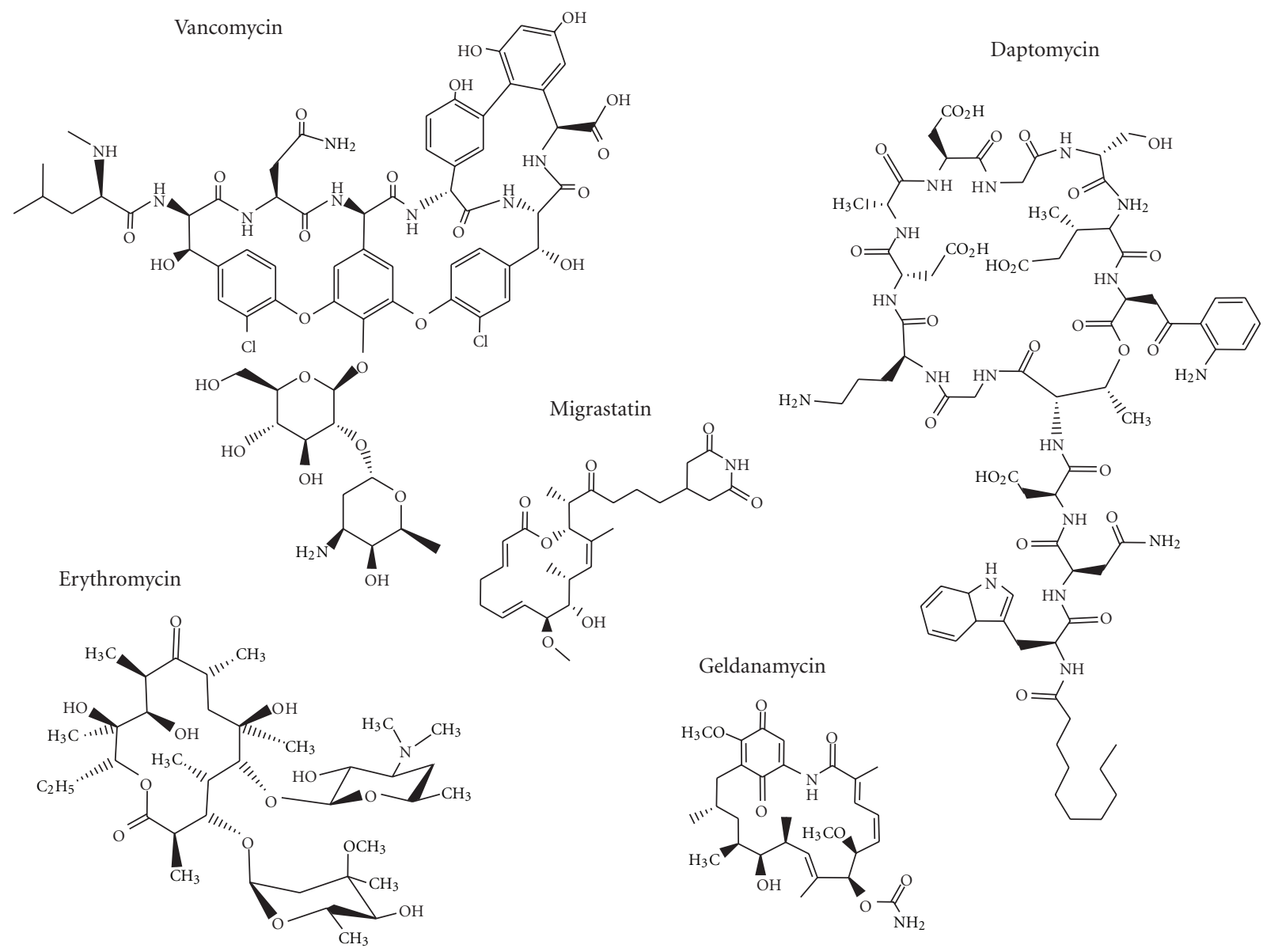

FIGURE 1: Examples of natural compounds produced by actinomycetes. Vancomycin and daptomycin are synthesized by NRPSs from Amycolatopsis orientalis and Streptomyces roseosporus, respectively; erythromycin, migrastatin, and meridamycin are produced by PKSs in Saccharopolyspora erythraea, Streptomyces platensis, and Streptomyces sp. NRRL 30748.

original producer to a host with well-defined genetics and physiology, where the genes can be expressed. Heterologous expression of large biosynthetic pathways can also be necessary in all cases in which producing bacteria are not cultivable or to examine new metabolites produced by cryptic gene clusters, usually revealed by genome sequencing and mining. This paper deals with artificial chromosome-based vectors and related methods currently available to analyze biosynthetic genes and to express biosynthetic pathways in heterologous systems.

\section{Handling Large Actinomycete DNA Fragments: Construction of E. coli-Streptomyces Artificial Chromosomes}

The first step for studying and analyzing biosynthesis at the genetic level is to obtain gene sequences. The recent genomic technologies allow to sequence entire genomes and thereby to identify biosynthetic gene clusters [6]. However, the majority of natural-product-producing microorganisms are refractory to standard gene manipulation techniques, and some are not cultivatable outside their ecological context
[7, 8]. For these reasons, the study and modification of whole pathways has greatly benefited from the possibility to manipulate them in vectors, such as cosmids, and artificial chromosomes. Cosmids show high transformation efficiency, with a loading capacity up to $45 \mathrm{~kb}$. Many cosmid libraries of actinomycetes have been successfully constructed to clone and identify biosynthetic gene clusters [9-14]. However, many biosynthetic gene clusters for natural products are larger than the average insert size of common cosmid vectors. To overcome this size limitation, one strategy can be the subcloning of subsets of the biosynthetic gene cluster into compatible expression plasmids followed by their introduction and coexpression in a suitable host strain $[15,16]$. Another option is the reassembly of large natural product pathways on a single transferable vector by using the recombineering (recombination-mediated genetic engineering) technology that employs homologous recombination mediated by phage-based recombination systems. Recombineering is independent of the E. coli endogenous homologous recombination functions, of restriction sites and of DNA size. Recombineering using the E. coli $\lambda$ phage Red pathway, which is mediated by its Exo and Beta proteins, was successfully applied to reconstitute phenalinolactone and 
anthramycin gene clusters from two independent cosmids $[17,18]$ in E. coli.

For a single step cloning of inserts larger than $100 \mathrm{~kb}$ a more straightforward strategy uses bacterial artificial chromosome (BAC), based on the E. coli F factor, and the P1derived artificial chromosome (PAC), which can carry large DNA fragments (about 100-300 kb) in E. coli cells [19]. E. coli is not suitable to heterologously express actinomycete genes which are $\mathrm{G}+\mathrm{C}$ rich [20]. Thus, vectors that can be shuttled between E. coli, where library construction and manipulation can be easily conducted, and Streptomyces, where actinomycete genes can be expressed (Figure 2), were developed [21-23].

So far, three E. coli-Streptomyces artificial chromosomes have been constructed. The features of pPAC-S2 (derived from pCYPAC2 [21]), pSTREPTOBAC $\mathrm{V}$ (derived from pBACe3.6 [22]), and pSBAC (derived from pCC1BAC [23]) are briefly reported in Table 1 and maps are given in Figure 3. All of them are replicative in E. coli and integrative in Streptomyces. Integrative vectors are maintained in the host without selection and are more stable, since metabolic burden and unwanted recombination events are reduced.

Integration into actinomycete chromosome is ensured by the attP-int system derived from the temperate phages $\Phi C 31$ or $\Phi B T 1$, which directs the integration of the vector DNA at the corresponding chromosomal attB sites of the host. The availability of two compatible integrating artificial chromosomes can increase the possibility to further manipulate the host. A detrimental effect on endogenous antibiotic production was reported in some producer strains after the integration of vectors in the $\Phi C 31$ attB site. For example, the integration of nonrecombinant vectors of $11 \mathrm{~kb}$ and $48 \mathrm{~kb}$ caused a 90 and $59 \%$ reduced production of the endogenous glycopeptide A47934 in Streptomyces toyocaensis [24-26].

In addition, vectors contain a resistance marker to select recombinant clones. Some antibiotic resistance cassettes, like ampicillin resistance gene, are not useful for selection in Streptomyces and different resistance cassettes can be necessary for selection in the two hosts. As an example, the pPACS2 contains kanamycin and thiostrepton resistance cassettes used for selecting E. coli and Streptomyces recombinant clones, respectively. pSTREPTOBAC V and pSBAC contain an apramycin resistance cassette that can be used to select both E. coli and Streptomyces recombinant clones.

To transfer BACs or PACs from E. coli to Streptomyces two main strategies can be adopted. In pSTREPTOBAC $\mathrm{V}$ and pSBAC, an oriT in the vector allows intergeneric conjugation between an E. coli strain containing Tra encoding genes (carried in an helper plasmid [7] or into the chromosome [27]) and Streptomyces. When oriT is not present (i.e., pPAC-S2), a more laborious yet easy and well-established protocol is necessary, which comprises protoplast preparation and PEG-assisted protoplast transformation [7].

\section{Actinomycete Genomic Libraries Constructed in Artificial Chromosomes}

To date, several libraries have been constructed in BAC or PAC artificial chromosomes [22, 23, 28-32] and relevant features of these libraries are mentioned in Table 2.
Using Sau3AI, libraries of S. coelicolor and Planobispora rosea with an average insert size of $60 \mathrm{~kb}$ were constructed in pPAC-S2 [28]. A partial genomic library (two-fold coverage) of $S$. coelicolor was first constructed to set up the methodologies, and using the same procedure the first PAC library of an intractable actinomycete, $P$. rosea, with an average insert size of $60 \mathrm{~kb}$ and the largest insert of $150 \mathrm{~kb}$ was constructed. When the same methodology was applied to Nonomuraea sp. ATCC 39727, it was not successful, since its DNA undergoes degradation during PFGE $[29,37]$. The PFGE step was therefore omitted, and a high-quality, high molecular weight genomic library of 2,051 recombinant clones with inserts larger than 30 up to $155 \mathrm{~kb}$ with an average insert size of $57 \mathrm{~kb}$ was constructed in PPAC-S2.

To construct genomic libraries of $S$. roseosporus and S. platensis, Bam HI digested genomic DNA was ligated to BamHI digested pSTREPTOBAC V. Libraries of 2000 and 2400 clones with an average insert size of 71 and $75 \mathrm{~kb}$ were obtained from S. roseosporus and S. platensis $[22,31]$. The use of the frequently cutting Sau3AI allows a more random selection of inserts. BamHI, on the other hand, allows to obtain large inserts due to its less frequent restriction pattern. The BamHI-digested S. platensis DNA was fractionated twice by PFGE to reduce the number of small inserts.

Since many genome sequences are nowadays available, more specific approaches can be applied. In the case of Streptomyces sp. NRRL 30748, gene sequence analysis had already revealed that two MfeI sites flanked the $90 \mathrm{~kb}$ gene cluster devoted to meridamycin biosynthesis and no $\mathrm{MfeI}$ sites were present inside the gene cluster. Thus, genomic DNA was digested with $M f e I$ and resulting DNA fragments of approximately $100 \mathrm{~kb}$ were ligated to EcoRI sites of pSBAC [23].

If the biosynthetic gene cluster was cloned in a BAC/PACderived vector that does not contain the integration site for Streptomyces, the vector can be provided with an integration cassette and a marker for selection in Streptomyces, as in the case of the library of Streptomyces fradiae NRRL 18160 constructed in pECBAC. After the identification of a clone containing the gene cluster devoted to A54145 biosynthesis, the clone was modified and used to transform streptomycetes by adding the $\Phi \mathrm{C} 31$ attP-int cassette, the apramycin resistance cassette and oriT [30].

\section{Heterologous Expression of Biosynthetic Gene Clusters from Actinomycetes}

Heterologous expression of secondary metabolic gene clusters can allow process development and molecular biological manipulation of the biosynthetic pathways in those cases of native producer strains recalcitrant to manipulation. Even if this strategy appears effective and straightforward, choice of an appropriate host is always challenging.

E. coli is not a good host for the expression of actinomycete genes, and heavy manipulation has to be carried out to get biosynthesis of actinomycete products. As an example, to obtain erythromycin biosynthesis, the E. coli BL21 (DE3) strain containing the gene for T7 RNA polymerase was 

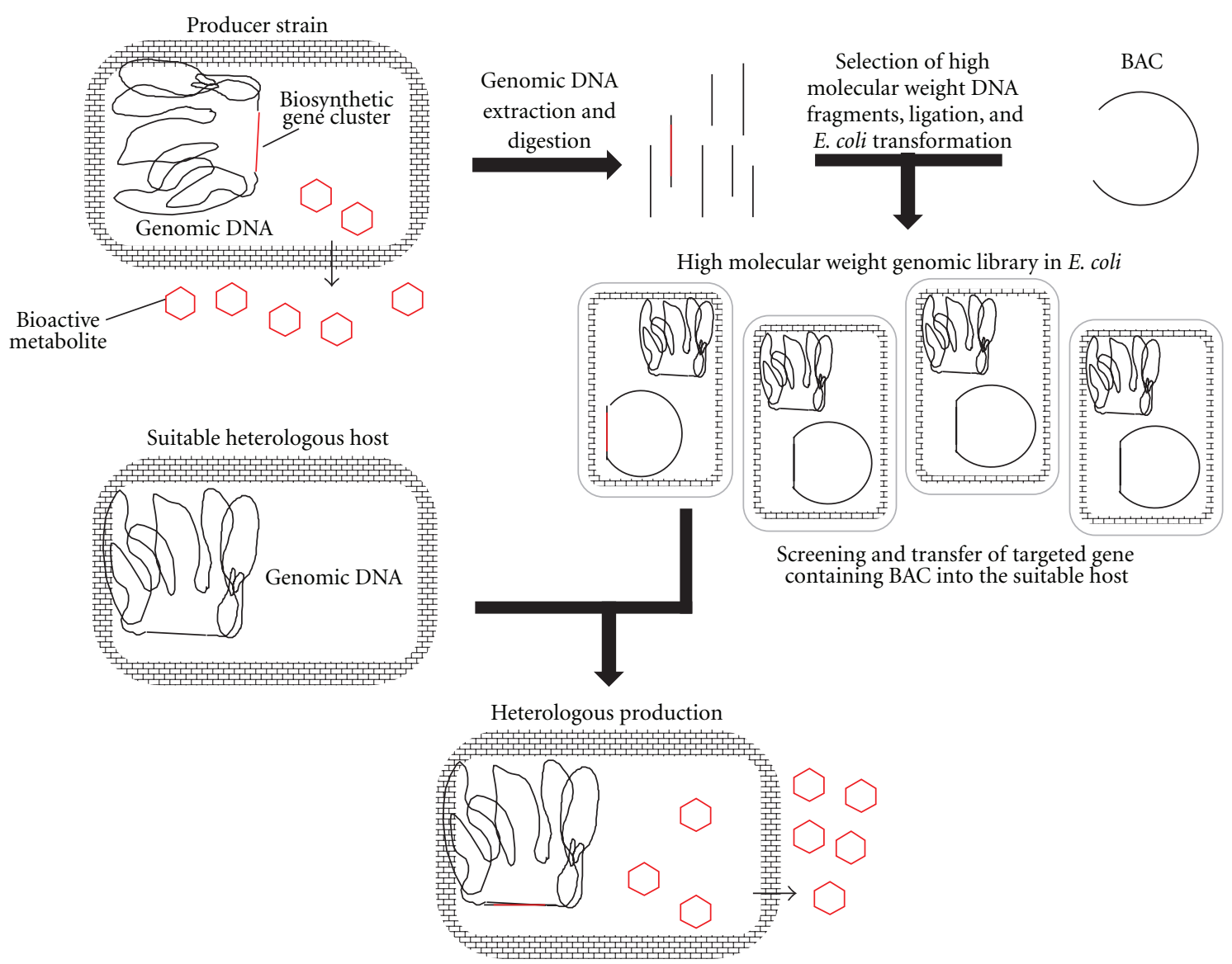

Figure 2: Generalized workflow for heterologous production of natural compounds in an amenable host. A high molecular weight genomic library is constructed and screened to identify one or two clones containing the biosynthetic gene cluster of interest. Once the clone is identified and sequenced to confirm the presence of all the genes, the recombinant vector DNA is used to transform the amenable host. Last, the heterologous production can be improved by changing fermentation process or by genetically manipulating the host.

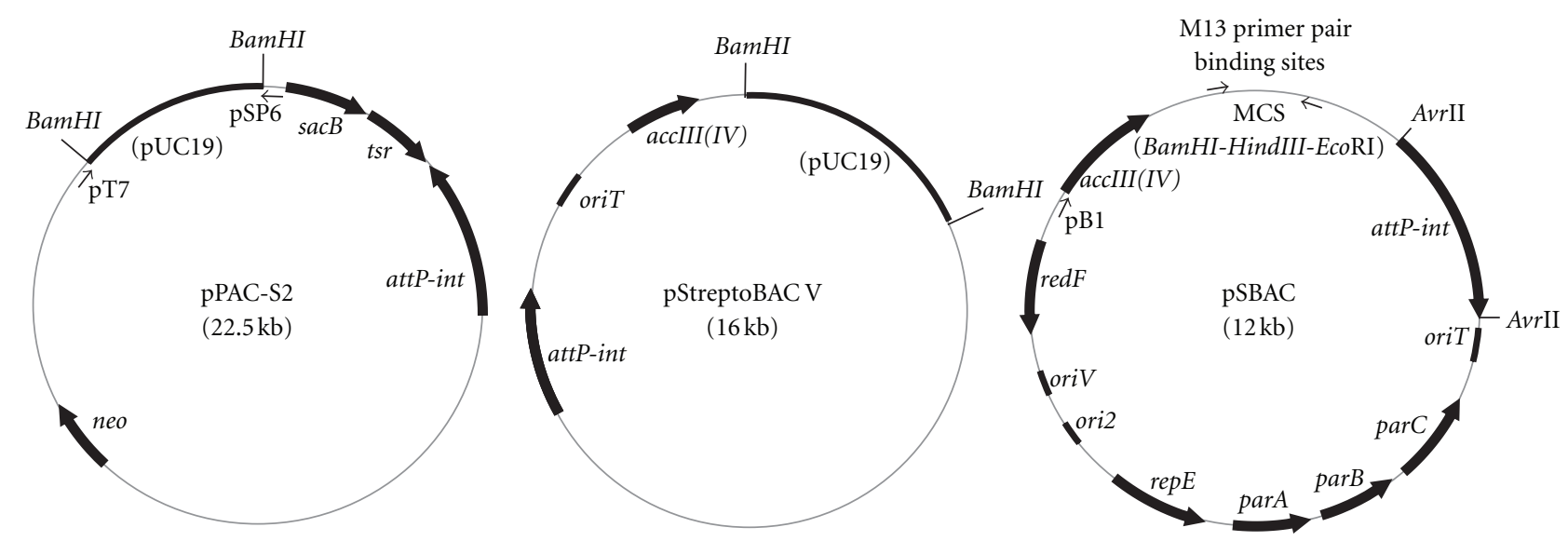

FIGURE 3: Comparison between physical maps of E. coli-Streptomyces artificial chromosomes. The vector pPAC-S2 derives from PAC, while pSTREPTOBAC V and pSBAC from BAC. The main features of the vectors are depicted: the resistance genes (neo, accIII(IV), tsr conferring resistance to kanamycin, apramycin, thiostrepton), attP-int cassettes for the site-specific integration in Streptomyces, restriction sites (BamHI, HindIII, EcoRI), sacB (conferring sensitivity to sucrose when expressed), oriT and oriV, devoted to intergeneric conjugation and inducible high copy number of the vector, respectively. 
TABLE 1: Shuttle E. coli-Streptomyces artificial chromosomes.

\begin{tabular}{lcccc}
\hline Vector & Derived from & attP-int cassette derived from & Resistance to & Reference \\
\hline pPAC-S2 & pCYPAC2 & C 31 & Kanamycin/thiostrepton in E. coli/Streptomyces & {$[21]$} \\
pSTREPTOBAC V & pBACe3.6 & DC31 & Apramycin in E. coli/Streptomyces & {$[22]$} \\
pSBAC & pCC1BAC & $\varphi B T 1$ & Apramycin in E. coli/Streptomyces & {$[23]$} \\
\hline
\end{tabular}

TABLE 2: Actinomycetes genomic libraries constructed in artificial chromosomes.

\begin{tabular}{lccccc}
\hline Source & Genome coverage & Average insert size & Genomic DNA digested with & Vector & Reference \\
\hline Planobispora rosea ATCC 53773 & $5 \mathrm{X}$ & $60 \mathrm{~kb}$ & Sau3AI & pPAC-S2 & {$[28]$} \\
Nonomuraea sp. ATCC 3972 & $5 \mathrm{X}$ & $57 \mathrm{~kb}$ & Sau3AI & pPAC-S2 & {$[29]$} \\
S. roseosporus NRRL 11379 & $17 \mathrm{X}$ & $71 \mathrm{~kb}$ & BamHI & pSTREPTOBAC V & {$[22]$} \\
S. platensis NRRL18993 & $22 \mathrm{X}$ & $75 \mathrm{~kb}$ & BamHI & pSTREPTOBAC V & {$[31]$} \\
Streptomyces sp. NRRL 30748 & $\mathrm{NR}$ & $\mathrm{NR}$ & MfeI & pSBAC* & {$[23]$} \\
S. autolyticus JX-47 & $50 \mathrm{X}$ & $150 \mathrm{~kb}$ & Sau3AI & pIndigoBAC5* & {$[32]$} \\
\hline
\end{tabular}

NR: not reported.

${ }^{*}$ Not integrative in Streptomyces.

transformed with four plasmids: (i) pHZT1 and (ii) pHZT2 containing all ery genes organized in two operons under T7 promoter control; (iii) pHZT4 containing eryK, the last gene of one of the operons, since it was not expressed at high level; and (iv) plasmid pGro7 containing the genes for the E. coli GroEL/ES chaperone system to aid protein folding and/or association of actinomycete gene products. Only the strain carrying the four plasmids produced erythromycin A, while the same E. coli strains containing only pHZT1 and pHZT2 did not. This very labour-intensive, time-consuming procedure was specific for erythromycin [38], and a similar strategy could not always be applied for the synthesis of other antibiotic compounds that may require "specific" precursors that E. coli cannot provide.

Streptomycetes are naturally the preferred hosts of actinomycete genes. Compared to other actinomycetes, Streptomyces is more amenable for strain improvement, grows more rapidly, and generally has the biosynthetic apparatus and primary precursors necessary to support natural product synthesis from exogenous pathways. Among streptomycetes, $S$. coelicolor and S. lividans have been widely used as heterologous hosts.

S. coelicolor is considered the model species among actinomycetes and a large array of genetic tools, including its genome sequence, is available to understand and manipulate the organism [7,39]. Although there are many advantages of using S. coelicolor as a host, plasmid-free derivatives of $S$. lividans, a close relative of $S$. coelicolor, have often been used for heterologous production (Table 3 ) [40, 41]. The primary reason is the absence of a strong restriction system, like in $S$. coelicolor, which heavily restricts methylated DNA [42].

Other streptomycetes have already been used for heterologous expression of secondary metabolite gene clusters, like Streptomyces albus G J1074 [35], and other industrial strains, such as S. avermitilis. S. albus G J1074 is a mutant defective in SalI restriction and modification, readily transformable cloning host that has been used for the expression of several other secondary metabolite gene clusters [35]. The industrial microorganism S. avermitilis, producer of the anthelmintic macrolide avermectins, has been already optimized for the efficient supply of primary metabolic precursors and biochemical energy to support multistep biosynthesis [43, 44].

4.1. Optimization of Heterologous Production in Streptomycetes. In some cases heterologous production is either absent or so low that manipulation of the host is necessary. Different strategies, both genetics- and physiology-driven, can be explored in a well-defined host. Fermentationbased approaches can consist of varying carbon or nitrogen sources or different phosphate levels in the medium and in feeding with a biosynthetic precursor that may be limiting during the growth phase. Genetic methods can target both host and cloned genes. Genomic analysis of streptomycetes has revealed that these microorganisms have large linear chromosomes that harbour over 20 gene clusters for secondary metabolites that could compete for precursors or could interfere with chemical analysis. Thus, the deletion of host gene clusters devoted to biosynthesis of other endogenous metabolites can increase precursor supply and, thus, improve heterologous production $[33,34,36$, $45,46]$; in addition, there are point mutations in $r p o B$ and $r p s L$ genes, coding for RNA polymerase beta-subunit and $30 \mathrm{~S}$ ribosomal subunit protein $\mathrm{S} 12$, respectively, known for increasing antibiotic production $[45,47]$. Although the underlying molecular mechanism is not understood, these mutations have a pleiotropic effect on levels of secondary metabolite production that is achieved, at least in part, by elevated transcript levels.

S. coelicolor produces at least four metabolites with antibiotic activity: the Type II polyketide actinorhodin (Act), the NPRS/PKS-derived prodiginines (Red), the NRPSderived calcium-dependent antibiotic (CDA), and the TypeI polyketide yellow pigment CPK [39]. In the last two years, two groups put their efforts in the engineering of $S$. coelicolor 
TABLE 3: Streptomycetes hosts.

\begin{tabular}{|c|c|c|}
\hline Strain & Genotype & Reference \\
\hline S. lividans TK23 & $s p c^{-} \mathrm{SLP}^{-} \mathrm{SLP3}^{-}$ & [7] \\
\hline S. lividans TK24 & str6 $^{-} \mathrm{SLP}^{-}{ }^{-} \mathrm{SLP}^{-}$ & {$[7]$} \\
\hline S. lividans TK64 & pro-2 str-6 SLP2- SLP3- & {$[7]$} \\
\hline S. lividans K4-114 & pro-2 str-6SLP2 ${ }^{-} S L P 3^{-} \triangle a c t:: e r m E$ Streptomyces & {$[33]$} \\
\hline S. coelicolor M512 & $\Delta r e d D \Delta$ actII-ORF4 SCP1 $1^{-} \mathrm{SCP} 2^{-}$ & {$[34]$} \\
\hline S. albus G J1074 & $i l v-1$ sal-2 & {$[35]$} \\
\hline S. avermitilis SUKA4/SUKA5 & $\Delta($ gapl-ptlL $):: e r m E(\Delta 3,745,502-3,758,936 \mathrm{nt})$ & {$[36]$} \\
\hline
\end{tabular}

in order to construct suitable production hosts $[45,46]$. In one case four of these endogenous secondary metabolite gene clusters were deleted and point mutations in $r p o B$ and rpsL genes were introduced [45]. In the more recent case, sequential deletions of all ten polyketide synthase (PKS) and nonribosomal peptide synthetase (NRPS) biosynthetic gene clusters and a 900-kb subtelomeric sequence (total ca. $1.22 \mathrm{Mb}, 14 \%$ of the genome) from S. coelicolor chromosome were performed to construct derivative strains [46]. In addition to lacking antibacterial activity, the engineered strains possess relatively simple extracellular metabolite profiles and they could markedly facilitate the discovery of new compounds by heterologous expression of cloned gene clusters, particularly the numerous cryptic secondary metabolic gene clusters that are prevalent within actinomycete genome sequences $[45,46]$.

S. lividans has been largely used as heterologous host for its more relaxed restriction/modification system [42], but some strains of $S$. lividans under many fermentation conditions produce actinorhodin that interferes with the detection and purification of other secondary metabolites, so part of the act gene cluster was deleted in K4-114, therefore providing a cleaner background for heterologous expression [33].

The industrial microorganism S. avermitilis was manipulated by deletion of the telomeric ends of the linear chromosome, which contain nonvital genes, often antibiotic biosynthesis-related [36]. A region of more than $1.4 \mathrm{Mb}$ was deleted stepwise from the $9.02 \mathrm{Mb}$ S. avermitilis linear chromosome to generate a series of defined deletion mutants (i.e., SUKA4, SUKA5), that did not produce any of the major endogenous secondary metabolites found in the parent strain.

Also foreign genes can be manipulated, that is, strong promoters can be added to drive transcription of genes or operons, otherwise weakly transcribed.

Here we discuss the successful production of daptomycin (from $d p t$ genes), meridamycin (from mer genes), and migrastatin (from $m g s$ genes), pointing out the strategies applied (Figure 4). Firstly, artificial chromosome libraries from S. roseosporus NRRL 11379, S. platensis NRRL18993, and Streptomyces sp. NRRL 30748 were screened by PCR or hybridization for the presence of $d p t, m g s$, mer, and $l p t$ gene clusters, respectively. Once sequenced, the relevant clones were used to transform various Streptomyces hosts, where they were stably integrated and maintained.
4.1.1. Production of Daptomycin in S. lividans. The daptomycin gene cluster was transferred in S. lividans TK64 and TK23. In both cases, the recombinant strains produced the lipopeptide with a low titre and a high amount of actinorhodin [22, 41]. To reduce the background effect of native metabolites, the $S$. lividans TK23 host was manipulated by inactivating actinorhodin production through replacement of a portion of the gene cluster with a resistance gene, but this did not result in a yield improvement. An improvement in daptomycin biosynthesis was obtained by increasing the levels of $\mathrm{K}_{2} \mathrm{HPO}_{4}$ during fermentation. It is likely that phosphate, often reported as an inhibitor of antibiotic production, inhibited host metabolite biosynthesis pathways, whose precursors could be necessary for daptomycin biosynthesis. Adjusting the level of phosphate in the medium the yield of the lipopeptide was increased from 20 to $55 \mathrm{mg} / \mathrm{L}$, corresponding to one-third of the yield of the natural producer $[22,41]$.

4.1.2. Production of Meridamycin in S. lividans. In the case of meridamycin biosynthesis, the transfer of the $90 \mathrm{~kb}$ gene cluster to S. lividans TK24 and K4-114 did not lead to the detection of any heterologous metabolites, since PKS genes were not properly transcribed, possibly due to the size of operon (3 genes for a $78 \mathrm{~kb}$ operon). Indeed, the cloning of a strong promoter (ermE promoter) upstream of the mer genes coding for the PKS allowed the detection of approximately $100 \mu \mathrm{g} / \mathrm{L}$ of meridamycin in the fermentation extract. To further increase the yield, a fermentation strategy based on feeding with biosynthetic precursors was applied. The supply of ethylmalonyl-CoA, used as an extender unit in meridamycin biosynthesis, is a critical factor that limits the synthesis of some polyketides by heterologous hosts. In fact, when S. lividans containing mer gene cluster with PKS genes under ermE control, was supplemented with diethyl malonate, which is an effective precursor for ethylmalonylCoA, the production increased about 2 -fold $(240 \mu \mathrm{g} / \mathrm{L})$. On the other hand, feeding with pipecolic acid, another unique precursor for the biosynthesis of meridamycin, did not increase production [23].

4.1.3. Production of Migrastatin in Streptomycetes. To find the most suitable host to express the biosynthetic gene cluster for the production of the polyketide migrastatin of $S$. platensis NRRL18993, five Streptomyces hosts were selected: S. lividans K4-114, S. coelicolor M512, S. albus G J1074, and the two 


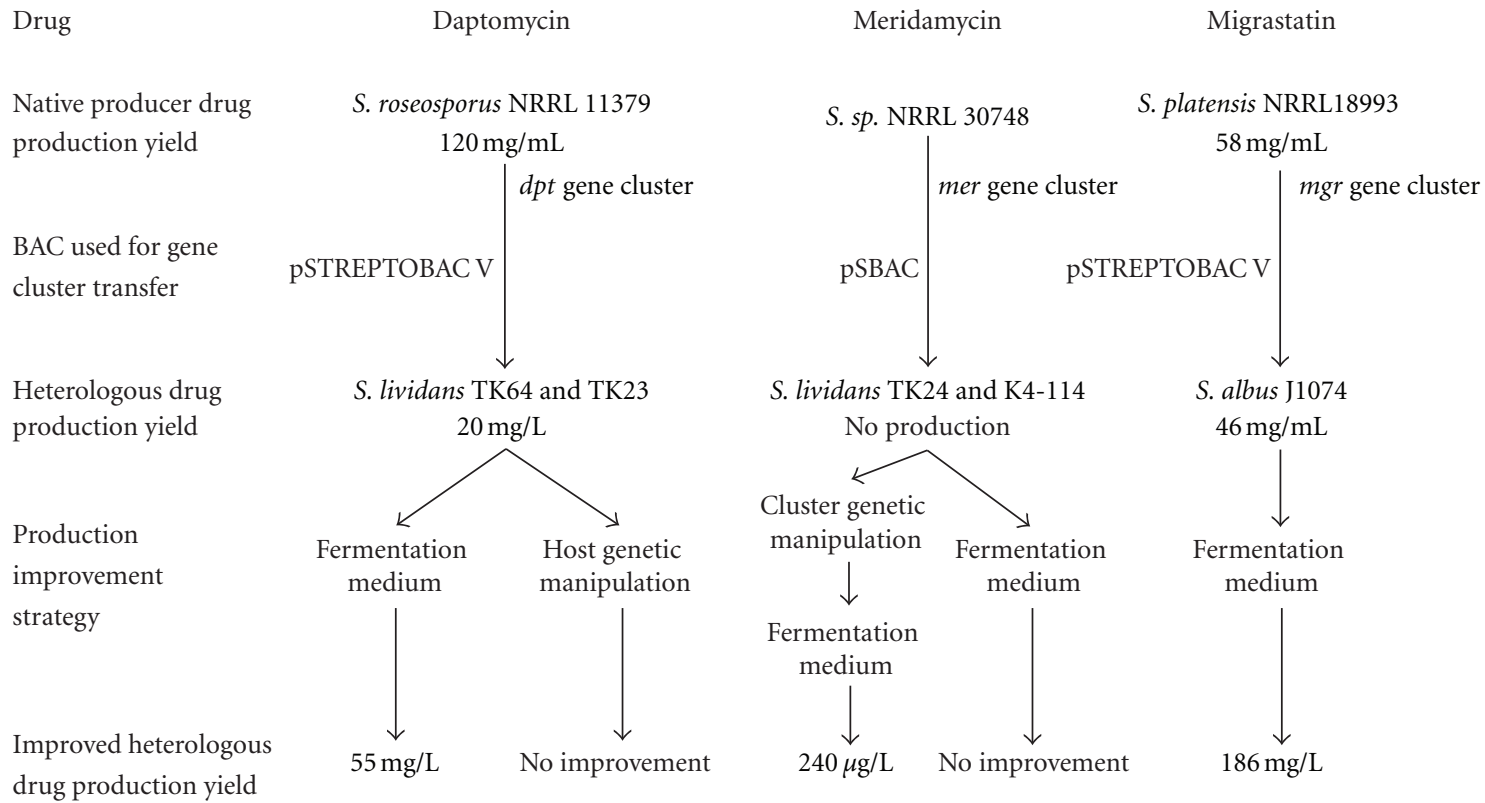

FIGURE 4: Strategies adopted to improve heterologous production of daptomycin, meridamycin, and migrastatin in heterologous hosts. Under "fermentation strategies" fall feeding with biosynthetic precursors or growth in presence of specific nutrients. Host genetic manipulation indicates the inactivation of host biosynthetic pathways or mutations in genes ( $r p s L$ and $r p o B)$ known for increasing secondary metabolite production. Genetic cluster manipulation refers to the cloning of a strong promoter upstream of foreign genes which are weakly transcribed. Details are provided in the text.

S. avermitilis strains, SUKA4 and SUKA5. All the five strains produced the metabolite, but the titres, in two media already optimized for the natural producer, were similar to or less than those obtained with S. platensis. The best producers were $S$. albus that produced approximately $46 \mathrm{mg} / \mathrm{L}$ in $\mathrm{B} 2$ medium (natural producer $58 \mathrm{mg} / \mathrm{L}$ ), and $S$. lividans $\mathrm{K} 4-114$ that produced $15 \mathrm{mg} / \mathrm{L}$ (control $17 \mathrm{mg} / \mathrm{L}$ ) in R2YED medium. Adjusting fermentation conditions, an improvement of the titer (appr. $186 \mathrm{mg} / \mathrm{L}$ ) was registered in S. albus. In addition, feeding with $\mathrm{NaHCO}_{3}$ that plays a critical role in the biosynthesis of malonyl-CoA, the major precursor for migrastatin biosynthesis, allowed S. albus to produce migrastatin at a titer of $213.8 \mathrm{mg} / \mathrm{L}$, about 5 -fold higher than the originally $[31,48]$.

4.1.4. Production of the Lipopeptide A54145 in S. ambofaciens and $S$. roseosporus. It is noteworthy that also the production of the lipopeptide A54145 from S. fradiae was obtained in streptomycetes hosts, but the vector, pECBAC1, was not an E. coli-Streptomyces shuttle vector. The recombinant vector, containing the entire lpt gene cluster was modified by adding the $\Phi C 31$ attP-int integration cassette after library construction [30]. The lpt gene cluster of $S$. fradiae was transferred into $S$. ambofaciens and $S$. roseosporus strains genetically manipulated in a such way to not being anymore able to produce spiramycin [49] and daptomycin [50], respectively. Interestingly, both sets of heterologous strains were more efficient than wild type $S$. fradiae at producing the lipopeptide, but less productive than the $S$. fradiae highproducer control.

\section{Future Perspectives}

Artificial chromosomes are useful tools to exploit or to explore the genetic potential of intractable actinomycetes, by creating, for example, large-insert libraries from strains of interest, in order to isolate one or more large gene clusters [22, 23, 28-32], or by reconstructing gene clusters that are already available as a set of smaller fragments cloned in lower capacity vectors $[17,18,51,52]$. To be transferred in amenable hosts, BAC and PAC are provided with means to be maintained in manipulable streptomycetes, which can synthesize foreign complex molecules, such as antibiotics [21-23]. Sometimes, heterologous expression of actinomycetes biosynthetic gene clusters was successful after changing fermentation conditions, that is, feeding with a biosynthetic precursor, minimizing background endogenous activities or after cloning strong promoters upstream of production genes weakly transcribed. The examples here described can be considered as promising starting points to successfully express heterologously valuable biosynthetic products. A significant improvement in heterologous production will surely come from new technologies, like next generation sequencing and gene stitching and synthesis. Indeed, the genome sequences of both the producing strains and of the hosts can help to find the minimal set of genes necessary to maintain heterologous production in a clean background and to avoid interference between foreign gene products and host that in some cases were reported $[53,54]$.

A possibility could be to create artificial hosts that maintain as less endogenous genes as possible and that contain the specific genes to provide primary metabolites and all the cofactors necessary to synthesize a specific secondary 
metabolite. Some studies in this direction have just started with the deletion of large DNA fragments from S. avermitilis and S. coelicolor chromosomes [36, 46]. More efforts are needed to obtain a universal suitable host of actinomycete gene clusters; although S. avermitilis and S. coelicolor strains with minimized genomes have been constructed and shown to produce more metabolites than natural producers, further manipulation of their genomes followed by the improvement of the yields will hopefully occur with the help of specific experiments of gene stitching and synthesis. The genome sequencing of Streptomyces species (e.g., S. coelicolor and $S$. avermitilis $[39,43])$ has remarkably revealed that each strain has the genetic information to produce a large number (e.g., 23 and 32, resp.) of secondary metabolites. This implies that as much as $90 \%$ of the chemical potential of these organisms remains undiscovered by the conventional screening programs. Genome mining offers a powerful method for tapping into these cryptic natural products [55]. For discovery of new compounds by genome mining, heterologous expression could be employed to examine new products by these new gene clusters.

Heterologous expression through artificial chromosomes may provide the possibility to access gene clusters for the production of bioactive metabolites from the large number of uncultured bacteria present in the environment without the need to actually isolate them [56-61]. Growing many of these organisms at large scale for production purposes is unlikely to be practical but their biosynthetic gene clusters are particularly attractive targets for heterologous expression in a genetically amenable host.

For all these cases, it will be important to develop a suite of streptomycete hosts for high-level heterologous expression of specific types of secondary metabolites, and to facilitate rapid identification and testing of novel products. Given the relatively recent success of heterologous complex natural product biosynthesis, these tools are just beginning to be applied, but they appear to be very promising. Such approaches, in addition to omics global characterization techniques and more traditional process engineering steps, will be of paramount importance for the maximization of natural product outputs from heterologous hosts.

When the new technologies (e.g., gene synthesis) will be cheap and there will be no more novel activities and genes to discover, it is likely that cloning strategy will be abandoned, but at the moment this perspective is quite far.

\section{Acknowledgments}

G. Gallo was supported by the European-funded project "LAntibiotic Production: Technology, Optimization and improved Process" (LAPTOP), KBBE-245066. The authors are very grateful to Dr. Luca Dolce for critical reading of and corrections on the paper.

\section{References}

[1] A. Raja and P. Prabakarana, "Actinomycetes and drug-an overview," American Journal of Drug Discovery and Development, vol. 1, no. 2, pp. 75-84, 2011.
[2] J. L. Adrio and A. L. Demain, "Genetic improvementof processes yielding microbial products," FEMS Microbiology Reviews, vol. 30, no. 2, pp. 187-214, 2006.

[3] R. H. Baltz, "Genetic methods and strategies for secondary metabolite yield improvement in actinomycetes," Antonie van Leeuwenhoek, vol. 79, no. 3-4, pp. 251-259, 2001.

[4] S. Donadio, P. Monciardini, R. Alduina et al., "Microbial technologies for the discovery of novel bioactive metabolites," Journal of Biotechnology, vol. 99, no. 3, pp. 187-198, 2002.

[5] Y. Mast, T. Weber, M. Gölz et al., "Characterization of the "pristinamycin supercluster" of Streptomyces pristinaespiralis," Microbial Biotechnology, vol. 4, no. 2, pp. 192-206, 2011.

[6] R. Kirby, "Chromosome diversity and similarity within the Actinomycetales," FEMS Microbiology Letters, vol. 319, no. 1, pp. 1-10, 2011.

[7] T. Kieser, M. J. Bibb, M. J. Buttner et al., Practical Streptomyces Genetics, The John Innes Foundation, Norwich, UK; Crowes, London, UK, 2000.

[8] J. Handelsman, "Metagenomics: application of genomics to uncultured microorganisms," Microbiology and Molecular Biology Reviews, vol. 68, no. 4, pp. 669-685, 2004.

[9] M. Steffensky, A. Mühlenweg, Z. X. Wang, S. M. Li, and L. Heide, "Identification of the novobiocin biosynthetic gene cluster of Streptomyces spheroides NCIB 11891," Antimicrobial Agents and Chemotherapy, vol. 44, no. 5, pp. 1214-1222, 2000.

[10] F. Pojer, S. M. Li, and L. Heide, "Molecular cloning and sequence analysis of the clorobiocin biosynthetic gene cluster: new insights into the biosynthesis of aminocoumarin antibiotics," Microbiology, vol. 148, no. 12, pp. 3901-3911, 2002.

[11] M. Sosio, S. Stinchi, F. Beltrametti, A. Lazzarini, and S. Donadio, "The gene cluster for the biosynthesis of the glycopeptide antibiotic A40926 by Nonomuraea species," Chemistry and Biology, vol. 10, no. 6, pp. 541-549, 2003.

[12] M. C. Cone, X. Yin, L. L. Grochowski, M. R. Parker, and T. M. Zabriskie, "The blasticidin S biosynthesis gene cluster from Streptomyces griseochromogenes: sequence analysis, organization, and initial characterization," ChemBioChem, vol. 4, no. 9, pp. 821-828, 2003.

[13] D. Singh, M. J. Seo, H. J. Kwon et al., "Genetic localization and heterologous expression of validamycin biosynthetic gene cluster isolated from Streptomyces hygroscopicus var. limoneus KCCM 11405 (IFO 12704)," Gene, vol. 376, no. 1-2, pp. 13-23, 2006.

[14] M. Koběrská, J. Kopecký, J. Olšovská et al., "Sequence analysis and heterologous expression of the lincomycin biosynthetic cluster of the type strain Streptomyces lincolnensis ATCC 25466," Folia Microbiologica, vol. 53, no. 5, pp. 395-401, 2008.

[15] Q. Xue, G. Ashley, C. R. Hutchinson, and D. V. Santi, "A multiplasmid approach to preparing large libraries of polyketides," Proceedings of the National Academy of Sciences of the United States of America, vol. 96, no. 21, pp. 11740-11745, 1999.

[16] L. Tang, S. Shah, L. Chung et al., "Cloning and heterologous expression of the epothilone gene cluster," Science, vol. 287, no. 5453, pp. 640-642, 2000.

[17] T. M. Binz, S. C. Wenzel, H. J. Schnell, A. Bechthold, and R. Müller, "Heterologous expression and genetic engineering of the phenalinolactone biosynthetic gene cluster by using red/ET recombineering," ChemBioChem, vol. 9, no. 3, pp. 447454, 2008.

[18] Y. Hu, V. V. Phelan, C. M. Farnet, E. Zazopoulos, and B. O. Bachmann, "Reassembly of anthramycin biosynthetic gene cluster by using recombinogenic cassettes," ChemBioChem, vol. 9, no. 10, pp. 1603-1608, 2008. 
[19] H. Shizuya, B. Birren, U. J. Kim et al., "Cloning and stable maintenance of 300-kilobase-pair fragments of human DNA in Escherichia coli using an F-factor-based vector," Proceedings of the National Academy of Sciences of the United States of America, vol. 89, no. 18, pp. 8794-8797, 1992.

[20] W. R. Strohl, "Compilation and analysis of DNA sequences associated with apparent streptomycete promoters," Nucleic Acids Research, vol. 20, no. 5, pp. 961-974, 1992.

[21] M. Sosio, F. Giusino, C. Cappellano, E. Bossi, A. M. Puglia, and S. Donadio, "Artificial chromosomes for antibiotic-producing actinomycetes," Nature Biotechnology, vol. 18, no. 3, pp. 343345,2000

[22] V. Miao, M. F. Coëffet-LeGal, P. Brian et al., "Daptomycin biosynthesis in Streptomyces roseosporus: cloning and analysis of the gene cluster and revision of peptide stereochemistry," Microbiology, vol. 151, no. 5, pp. 1507-1523, 2005.

[23] H. Liu, H. Jiang, B. Haltli et al., "Rapid cloning and heterologous expression of the meridamycin biosynthetic gene cluster using a versatile Escherichia coli-Streptomyces artificial chromosome vector, pSBAC," Journal of Natural Products, vol. 72, no. 3, pp. 389-395, 2009.

[24] R. H. Baltz, "Genetic manipulation of antibiotic-producing Streptomyces," Trends in Microbiology, vol. 6, no. 2, pp. 76-83, 1998.

[25] P. Matsushima, M. C. Broughton, J. R. Turner, and R. H. Baltz, "Conjugal transfer of cosmid DNA from Escherichia coli to Saccharopolyspora spinosa: effects of chromosomal insertions on macrolide A83543 production," Gene, vol. 146, no. 1, pp. 39-45, 1994.

[26] P. Matsushima and R. H. Baltz, "A gene cloning system for 'Streptomyces toyocaensis," Microbiology, vol. 142, no. 2, pp. 261-267, 1996.

[27] M. D. McMahon, C. Guan, J. Handelsman, and M. G. Thomas, "Metagenomics in Streptomyces lividans reveals hostdependent functional expression," Applied Environmental Microbiology, vol. 78, no. 10, pp. 3622-3629, 2012.

[28] R. Alduina, S. De Grazia, L. Dolce et al., "Artificial chromosome libraries of Streptomyces coelicolor A3(2) and Planobispora rosea," FEMS Microbiology Letters, vol. 218, no. 1, pp. 181-186, 2003.

[29] R. Alduina, A. Giardina, G. Gallo et al., "Expression in Streptomyces lividans of Nonomuraea genes cloned in an artificial chromosome," Applied Microbiology and Biotechnology, vol. 68, no. 5, pp. 656-662, 2005.

[30] D. C. Alexander, J. Rock, X. He, P. Brian, V. Miao, and R. H. Baltz, "Development of a genetic system for combinatorial biosynthesis of lipopeptides in Streptomyces fradiae and heterologous expression of the A54145 biosynthesis gene cluster," Applied and Environmental Microbiology, vol. 76, no. 20, pp. 6877-6887, 2010.

[31] Z. Feng, L. Wang, S. R. Rajski, Z. Xu, M. F. Coeffet-LeGal, and B. Shen, "Engineered production of iso-migrastatin in heterologous Streptomyces hosts," Bioorganic and Medicinal Chemistry, vol. 17, no. 6, pp. 2147-2153, 2009.

[32] S. Dai, Y. Ouyang, G. Wang, and X. Li, "Streptomyces autolyticus JX-47 large-insert bacterial artificial chromosome library construction and identification of clones covering geldanamycin biosynthesis gene cluster," Current Microbiology, vol. 63, no. 1, pp. 68-74, 2011.

[33] R. Ziermann and M. C. Betlach, "Recombinant polyketide synthesis in Streptomyces: engineering of improved host strains," BioTechniques, vol. 26, no. 1, pp. 106-110, 1999.

[34] B. Floriano and M. Bibb, "afsR is a pleiotropic but conditionally required regulatory gene for antibiotic production in
Streptomyces coelicolor A3(2)," Molecular Microbiology, vol. 21, no. 2, pp. 385-396, 1996.

[35] K. F. Chater and L. C. Wilde, "Streptomyces albus G mutants defective in the SalGI restriction-modification system," Journal of General Microbiology, vol. 116, no. 2, pp. 323-334, 1980.

[36] M. Komatsu, T. Uchiyama, S. Omura, D. E. Cane, and H. Ikeda, "Genome-minimized Streptomyces host for the heterologous expression of secondary metabolism," Proceedings of the National Academy of Sciences of the United States of America, vol. 107, no. 6, pp. 2646-2651, 2010.

[37] M. L. Beyazova, B. C. Brodsky, M. C. Shearer, and A. C. Horan, "Preparation of actinomycete DNA for pulsedfield gel electrophoresis," International Journal of Systematic Bacteriology, vol. 45, no. 4, pp. 852-854, 1995.

[38] H. Zhang, K. Skalina, M. Jiang, and B. A. Pfeifer, "Improved E. coli erythromycin a production through the application of metabolic and bioprocess engineering," Biotechnology Progress, vol. 8, no. 1, pp. 292-296, 2012.

[39] S. D. Bentley, K. F. Chater, A. M. Cerdeno-Tarraga et al., "Complete genome sequence of the model actinomycete Streptomyces coelicolor A3(2)," Nature, vol. 417, pp. 141-147, 2002.

[40] Z. Hu, D. A. Hopwood, and C. Khosla, "Directed transfer of large DNA fragments between Streptomyces species," Applied and Environmental Microbiology, vol. 66, no. 5, pp. 2274-2277, 2000.

[41] J. Penn, X. Li, A. Whiting et al., "Heterologous production of daptomycin in Streptomyces lividans," Journal of Industrial Microbiology and Biotechnology, vol. 33, no. 2, pp. 121-128, 2006.

[42] D. J. MacNeil, "Characterization of a unique methyl-specific restriction system in Streptomyces avermitilis," Journal of Bacteriology, vol. 170, no. 12, pp. 5607-5612, 1988.

[43] H. Ikeda, J. Ishikawa, A. Hanamoto et al., "Complete genome sequence and comparative analysis of the industrial microorganism Streptomyces avermitilis," Nature Biotechnology, vol. 21, no. 5, pp. 526-531, 2003.

[44] R. H. Baltz, "Streptomyces and Saccharopolyspora hosts for heterologous expression of secondary metabolite gene clusters," Journal of Industrial Microbiology and Biotechnology, vol. 37, no. 8, pp. 759-772, 2010.

[45] J. P. Gomez-Escribano and M. J. Bibb, "Engineering Streptomyces coelicolor for heterologous expression of secondary metabolite gene clusters," Microbial Biotechnology, vol. 4, no. 2, pp. 207-215, 2011.

[46] M. Zhou, X. Jing, P. Xie et al., "Sequential deletion of all the PKS and NRPS biosynthetic gene clusters and a 900-kb subtelomeric sequence of the linear chromosome of Streptomyces coelicolor," FEMS Microbiology Letters, vol. 333, no. 2, pp. 169-179, 2012.

[47] A. Talà, G. Wang, M. Zemanova, S. Okamoto, K. Ochi, and P. Alifano, "Activation of dormant bacterial genes by Nonomuraea sp. strain ATCC 39727 mutant-type RNA polymerase," Journal of Bacteriology, vol. 191, no. 3, pp. 805-814, 2009.

[48] D. Yang, X. Zhu, X. Wu et al., "Titer improvement of isomigrastatin in selected heterologous Streptomyces hosts and related analysis of mRNA expression by quantitative RT-PCR," Applied Microbiology and Biotechnology, vol. 89, no. 6, pp. 1709-1719, 2011.

[49] M. A. Richardson, S. Kuhstoss, M. L. B. Huber et al., "Cloning of spiramycin biosynthetic genes and their use in constructing Streptomyces ambofaciens mutants defective in spiramycin biosynthesis," Journal of Bacteriology, vol. 172, no. 7, pp. 3790 3798, 1990. 
[50] V. Miao, M. F. Coëffet-Le Gal, K. Nguyen et al., "Genetic engineering in Streptomyces roseosporus to produce hybrid lipopeptide antibiotics," Chemistry and Biology, vol. 13, no. 3, pp. 269-276, 2006.

[51] M. Sosio, E. Bossi, and S. Donadio, "Assembly of large genomic segments in artificial chromosomes by homologous recombination in Escherichia coli," Nucleic acids research, vol. 29, no. 7, p. E37, 2001

[52] O. Perlova, J. Fu, S. Kuhlmann et al., "Reconstitution of the myxothiazol biosynthetic gene cluster by red/ET recombination and heterologous expression in Myxococcus xanthus," Applied and Environmental Microbiology, vol. 72, no. 12, pp. 7485-7494, 2006.

[53] D. E. Gillespie, S. F. Brady, A. D. Bettermann et al., "Isolation of antibiotics turbomycin A and B from a metagenomic library of soil microbial DNA," Applied and Environmental Microbiology, vol. 68, no. 9, pp. 4301-4306, 2002.

[54] A. Giardina, R. Alduina, E. Gottardi, V. Di Caro, R. D. Süssmuth, and A. M. Puglia, "Two heterologously expressed Planobispora rosea proteins cooperatively induce Streptomyces lividans thiostrepton uptake and storage from the extracellular medium," Microbial Cell Factories, vol. 9, article 44, 2010.

[55] R. H. Baltz, "Renaissance in antibacterial discovery from actinomycetes," Current Opinion in Pharmacology, vol. 8, no. 5, pp. 557-563, 2008.

[56] O. Béjà, M. T. Suzuki, E. V. Koonin et al., "Construction and analysis of bacterial artificial chromosome libraries from a marine microbial assemblage," Environmental Microbiology, vol. 2, no. 5, pp. 516-529, 2000.

[57] A. Martinez, S. J. Kolvek, C. L. T. Yip et al., "Genetically modified bacterial strains and novel bacterial artificial chromosome shuttle vectors for constructing environmental libraries and detecting heterologous natural products in multiple expression hosts," Applied and Environmental Microbiology, vol. 70, no. 4, pp. 2452-2463, 2004.

[58] M. R. Rondon, P. R. August, A. D. Bettermann et al., "Cloning the soil metagenome: a strategy for accessing the genetic and functional diversity of uncultured microorganisms," Applied and Environmental Microbiology, vol. 66, no. 6, pp. 2541-2547, 2000.

[59] A. E. Berry, C. Chiocchini, T. Selby, M. Sosio, and E. M. H. Wellington, "Isolation of high molecular weight DNA from soil for cloning into BAC vectors," FEMS Microbiology Letters, vol. 223, no. 1, pp. 15-20, 2003.

[60] J. Singh, A. Behal, N. Singla et al., "Metagenomics: concept, methodology, ecological inference and recent advances," Biotechnology Journal, vol. 4, no. 4, pp. 480-494, 2009.

[61] Y. Ouyang, S. Dai, L. Xie et al., "Isolation of high molecular weight DNA from marine sponge bacteria for BAC library construction," Marine Biotechnology, vol. 12, no. 3, pp. 318325,2010 . 

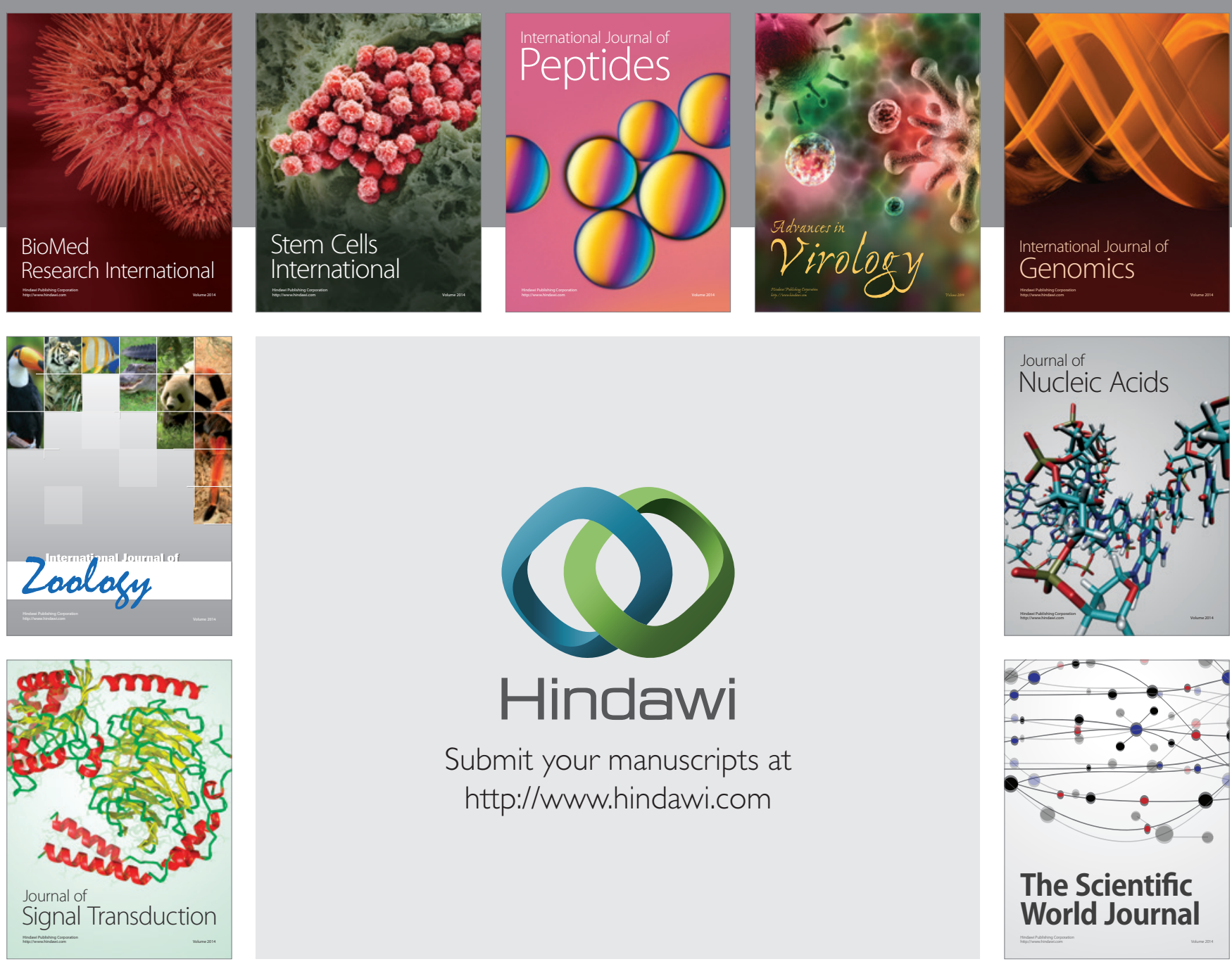

Submit your manuscripts at

http://www.hindawi.com
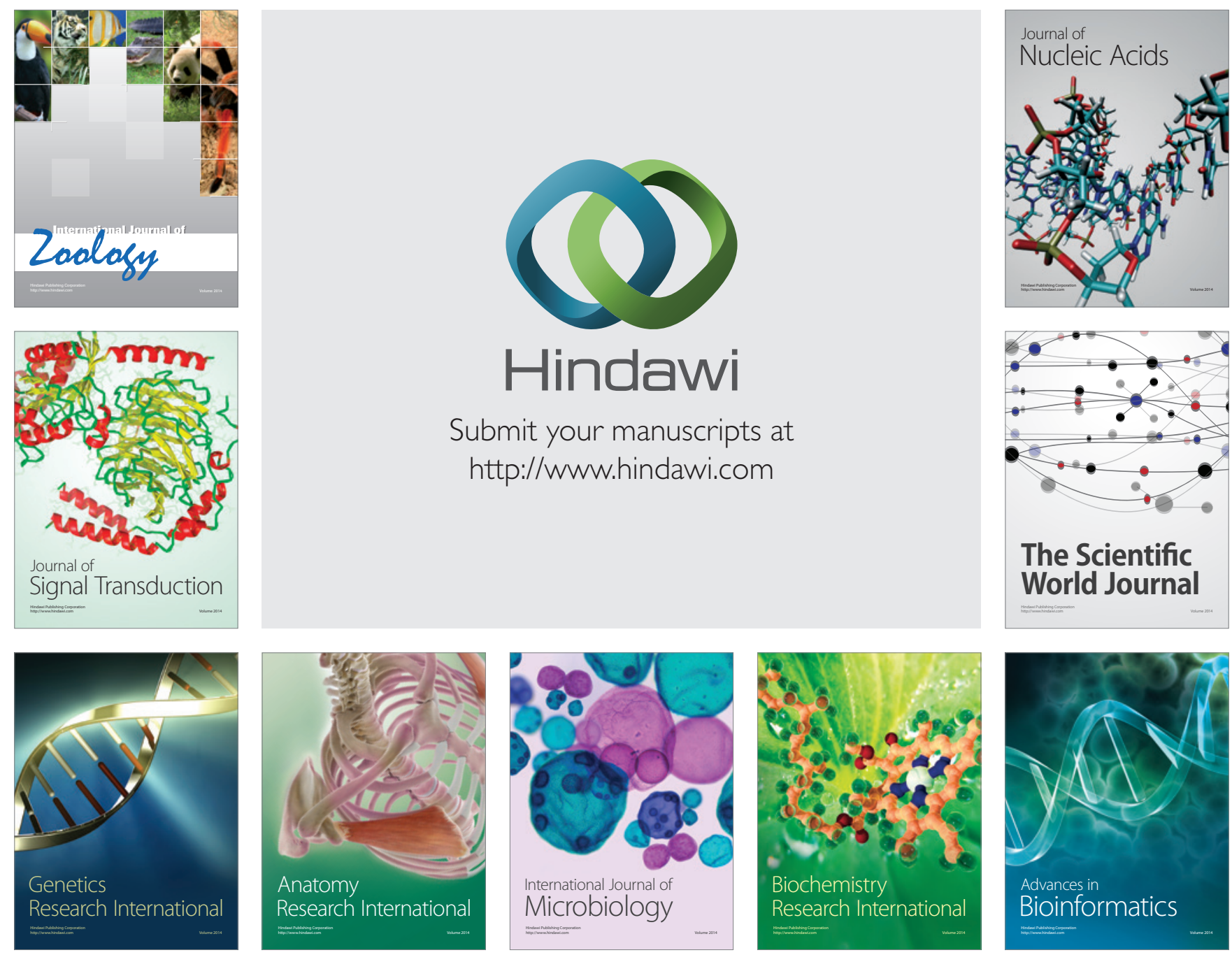

The Scientific World Journal
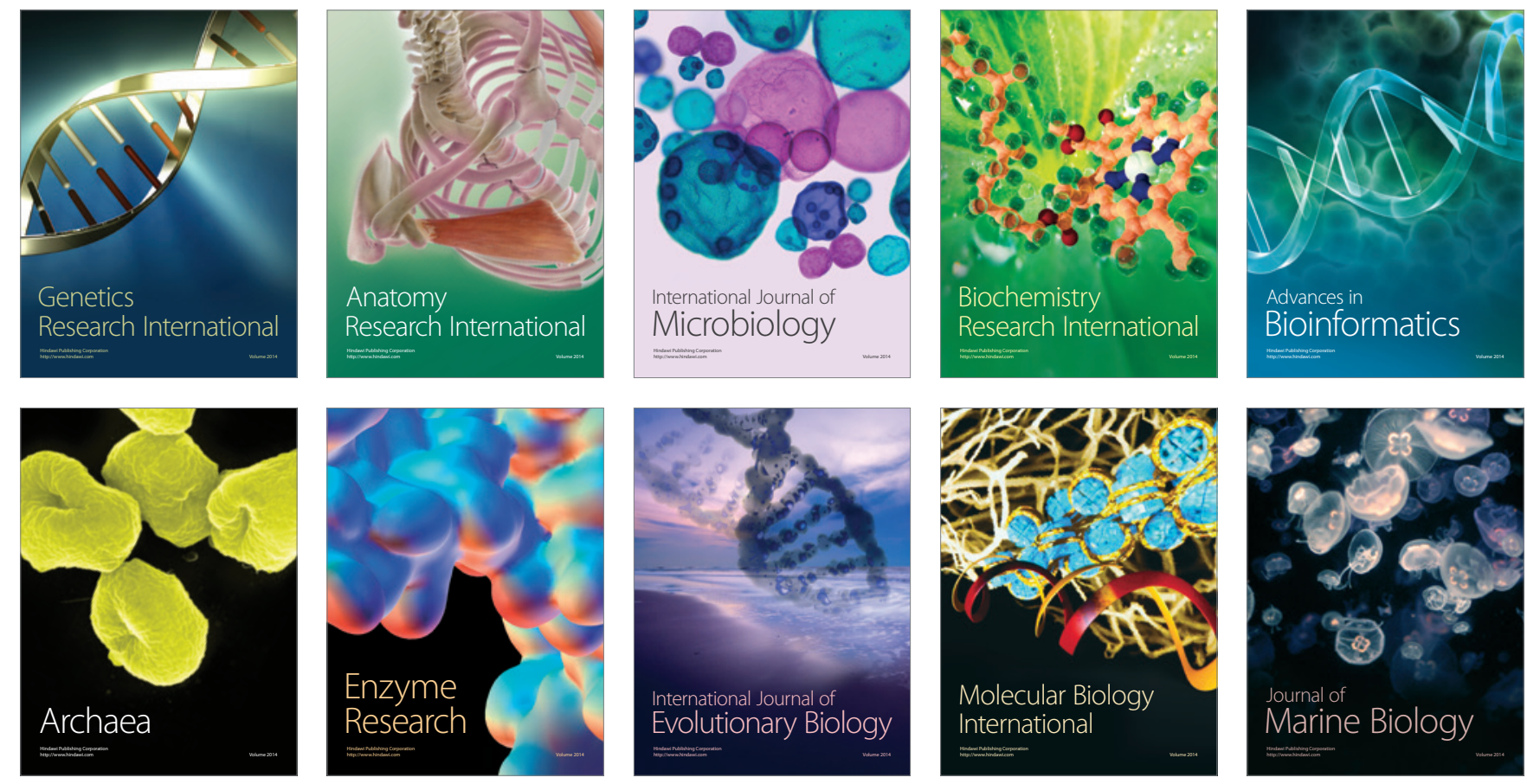